\title{
Image Processing of Artificial TARgets for Automatic Evaluation of Spray Quality
}

\author{
A. R. S. Marçal, M. Cunha
}

\begin{abstract}
A fully automatic methodology based on image processing is proposed to evaluate the quality of spray application sampled by water-sensitive papers (WSP). The methods proposed permit a computation of the fraction of spray coverage, an evaluation of the homogeneity of the spray spatial spread at various scales and directions, and extraction of stain and droplet size range and distribution. This allows the number of droplets per unit area and the standard droplet size spectra factors to be computed. The methods were tested with a number of test samples scanned at different resolutions, proving to be effective in situations where there is high spray coverage in the WSP, thus with considerable overlap between stains. The most suitable scanning resolution was found to be 600 dpi. The results obtained by the image processing methods were successfully compared with a manual (visual) counting of stains in a test sample.
\end{abstract}

Keywords. Homogeneity indices, Image processing, Spot size, Spray coverage, Water-sensitive paper.

$\mathbf{P}$ esticides and many fertilizers are often applied as liquid solutions, emulsions, and suspensions sprayed onto plant foliage or onto the soil. A proper adjustment of the sprayer improves the accuracy and the efficiency, resulting in a more uniform and targeted spray deposit, which contributes to preventing inadequate pest control or plant response; induction of resistance; economical loss of product, time and labor; and environmental pollution. It is therefore important to monitor the spray quality and quantity reaching target and off-target areas in order to improve pesticide spray application efficiency and to reduce environmental contamination.

Many field techniques have been developed for evaluating spray deposits and coverage, such as passive samplers (e.g., water-sensitive paper), active samplers (e.g., air samples and rotorods), and digital devices. Reviews of some of these techniques have been published, as well as the limiting factors for measurements (Turner and Huntington, 1970; Bateman, 1993; Fox et al., 1998; Panneton, 2002; Fox et al., 2003; Giles and Downey, 2003; Hoffman, 2003; Crowe et al., 2005 and Zhu et al. 2005).

Water-sensitive papers (WSP) have been used for more than 30 years (Turner and Huntington, 1970), being the most popular artificial targets for evaluation of spray coverage and deposit (Holownicki et al., 2002). Since water in spray stains the WSP and the spot size can be observed or measured, WSP can be used to evaluate the number of stains per unit area and

Submitted for review in April 2007 as manuscript number PM 6967; approved for publication by Power \& Machinery Division of ASABE in May 2008.

The authors are André R. S. Marçal, Assistant Professor, Faculdade de Ciências, Universidade do Porto, Portugal; and Mário Cunha, Assistant Professor, Secção Autónoma de Engenharia das Ciências Agrárias, Faculdade de Ciências, Universidade do Porto, Campus Agrário de Vairão, Portugal. Corresponding author: André R. S. Marçal, Faculdade de Ciências, Universidade do Porto, DMA, Rua do Campo Alegre 687, 4169-007 Porto, Portugal, phone: +351-220402273; fax: +351220402209; e-mail: andre.marcal@fc.up.pt. to measure the percent area covered (Syngenta, 2002). Droplet sizing is also possible when a proper spread factor or calibration equation has been developed for specific image processing systems to convert droplet WSP stains to their actual size (Syngenta, 2002, Giles and Downey, 2003; Hoffman, 2003). The WSP can be evaluated by visual estimate (Syngenta, 2002; Fox, et al., 2003), by counting the stains under a lens, or by automatic image analysis (e.g., Panneton, 2002; Fox et al., 2003). There are different software applications that produce indicators related to the spray quality based on image processing of scanned WSP, such as Optomax (Syngenta, 2002), DropletScan (Wolf, 2003), AgroScan (2008), and UTHSCSA ImageTool (2002). However, most of these software tools have not been specifically developed for this application and are therefore unable to properly evaluate the WSP. This is particularly noticed in situations where the volume of application is not sufficiently low to avoid considerable overlap between stain (Bateman, 1993; Fox et al., 1998; Salyani and Fox, 1999; Holownicki et al., 2002; Panneton, 2002; Fox et al., 2003). The current image-based analysis systems are neither effective nor accurate for measuring spot density in WSP when coverage is greater than about 30\% to $40 \%$ (Holownicki et al., 2002; Fox et al., 2003).

The WSP are available in different sizes and can be extremely useful when visualizing droplet sizes from different type of nozzles, droplet densities, spray distributions, and spray widths as well as penetration of spray into the crop canopy from aerial and ground spray applications. Droplet size spectra factors can be used to compare the behavior of different nozzles and as inputs for computer models to predict, for example, the dispersion and deposition of aerially released spray material in spray application experiments. However, WSP have a limited value for quantitative assessment of droplet size measurement of high spray application rates. Manual droplet counting using lenses is limited to a maximum of around 200 droplets per $\mathrm{cm}^{2}$, the equivalent of medium to coarse sprays (Syngenta, 2002), in which case there is minimal overlap of stains. In situations of high spray coverage with overlapping spots, the contrast between the stained 
(blue) and unstained area is not strong, and thus visual evaluation becomes difficult (Panneton, 2002; Fox et al., 2003; Holownicki et al., 2002). In these situations, the percent of area covered is the most reliable parameter that can be extracted (Salyani and Fox, 1999), and WSP can only be used for rough visual assessment of spray distribution and/or crop penetration.

The advance of digital imaging technology has increased the resolution capabilities for spray particle sizing and distribution analysis from different passive samplers. However, there are still difficulties in the evaluation of homogeneity on the card and for samples with droplet overlap. Image processing techniques based on mathematical morphology have been used for granulometry studies (Dougherty and Sand, 1995) and can be modified according to the specific requirements of the research described here.

The objective of this work is to present a fully automatic methodology based on image processing to evaluate the quality of spray application sampled by WSP. A set of algorithms and methods were developed for two distinct purposes: (1) to evaluate the homogeneity of the spray spatial spread, and (2) to estimate the stain size range and distribution in situations of low stain density and high spray coverage with overlap between stains.

\section{Material ANd Methods \\ Water-Sensitive Papers}

The water-sensitive paper (WSP) targets used in this study (76 × $26 \mathrm{~mm}$, TeeJet, Spraying Systems Co., Wheaton, Ill.) were collected as part of a sprayer evaluation study within a vineyard. These papers are yellow, and the surface turns dark blue when water droplets make contact and spread. Applications with water were made at 250 to $1000 \mathrm{~L}^{-1}$ volume rates. These rates were obtained by using 'Red' and 'Blue' Albuz ATR cone nozzles (Ceramiques Techniques Desmarquest, Evreux, France).

Three representative samples of spot density and homogeneity distribution along the card were used, as well as a blank WSP. The four WSP test samples (S0, S1, S2, and S3) were digitized in a photographic scanner (EPSON Perfection 4990 Photo). Four different scanning resolutions were used, resulting in 24-bit color images of $590 \times$ 396 pixels $(200$ dots per inch, dpi), $885 \times 594$ pixels (300 dpi), $1770 \times 1189$ pixels (600 dpi), and $3541 \times 2379$ pixels (1200 dpi). The four test samples at $200 \mathrm{dpi}$ are presented in figure 1 (top) as gray-scale versions of the original RGB color images obtained from the scanner.

\section{Image Segmentation}

The RGB (red green blue) color images were initially converted to binary images, where the stains appeared as 1 (white) and the background as 0 (black). This was done by an image classification / segmentation process. Initially, the red, green, and blue components were filtered individually, using a $3 \times 3$ median filter. The filtered RGB image was converted to the HSI (hue saturation intensity) color model, where the color component was decoupled from the intensity (Gonzalez and Woods, 2002). The Otsu thresholding method was then applied to the hue component, splitting the image in two sections. The maximum hue from each section was used to characterize the background and foreground by
So
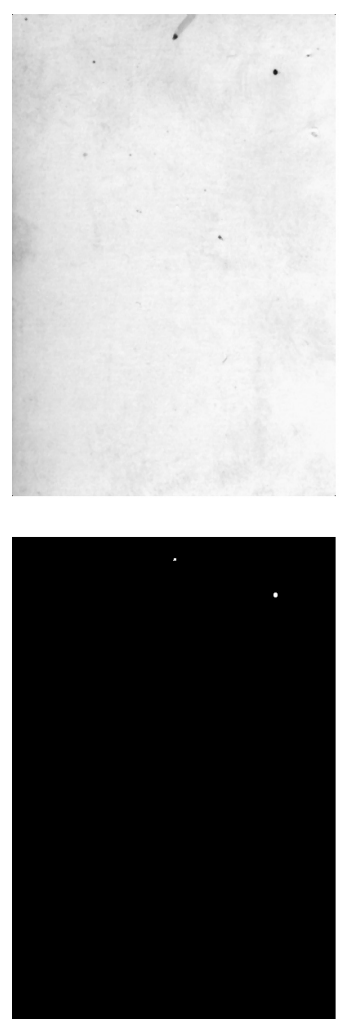

S1
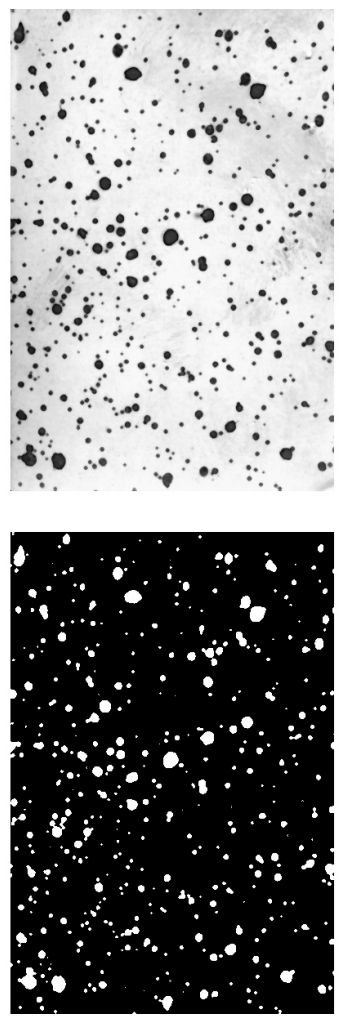

S2
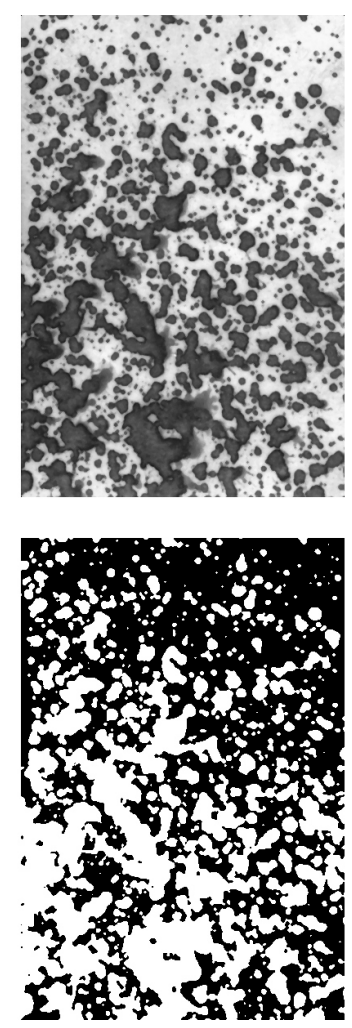

S3
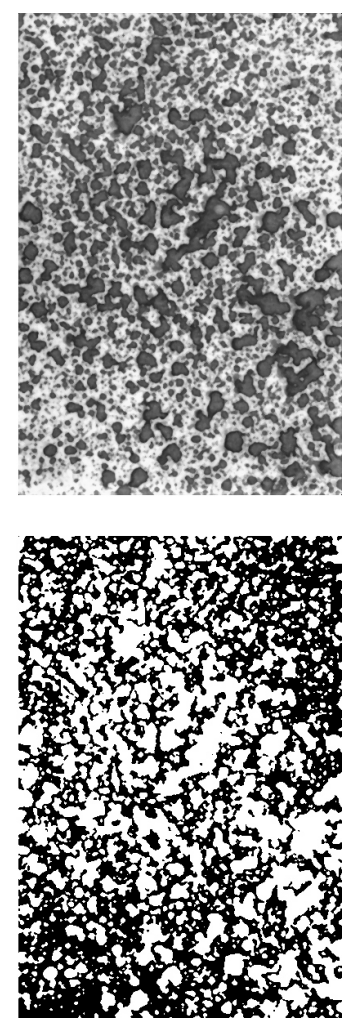

Figure 1. Gray-scale version of the original 24 bit RGB color test images (top row) and the corresponding binary images produced by the segmentation stage (bottom row). 

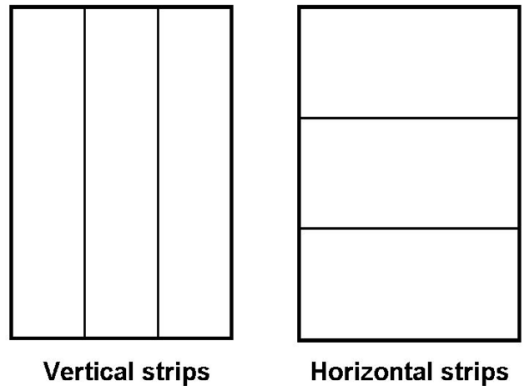

Horizontal strips

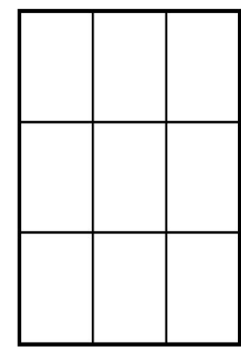

Sectors

Figure 2. Example of division of an image into $n$ strips, vertically (left) and horizontally (center), and into $n^{2}$ sectors (right), for $n=3$.

computing an average RGB vector for the background $\left(\mathrm{RGB}_{b}\right)$ and foreground $\left(\mathrm{RGB}_{f}\right)$. The RGB image was binarized using the Euclidian distance to the reference RGB vectors. Pixels with RGB values closer to $\mathrm{RGB}_{f}$ were considered as foreground (stain) and those closer to $\mathrm{RGB}_{b}$ as background. This process was tested with $100 \mathrm{WSP}$, with a success rate of $94 \%$. The six cases where the method failed were when the WSP was very heavily saturated. The resulting binary images for the four test samples are presented in figure 1 (bottom).

\section{HOMOGENEITY INDICES}

Direct counting of the foreground or spot pixels in the binary images (pixels with value 1 , or $\mathrm{ON}$ ) provides an estimation of the overall coverage of liquid in the WSP. It is also possible to evaluate the spatial homogeneity of the liquid on spread. This can be achieved using a new method established within this work, which is denoted as homogeneity indices, $H_{n}^{V}, H^{H}$, and $H_{n}^{S}$. The superscripts $V$, $H$, or $S$ represent vertical, horizontal, or sector, while the subscript $n$ represents a scale parameter (an integer not smaller than 2). The original image is divided into subimages by a factor of $n$. For example, for $n=3$, the image is divided into 3 vertical strips, 3 horizontal strips, and 9 (3 times 3) sectors, all of equal size, as illustrated in figure 2.

Let $B$ be a binary image and $B_{k l}$ a sub-image of $B$. The union of all $n^{2}$ sub-images $B_{k l}$ of $B$ results in image $B$ itself, as stated in equation 1 :

$$
B=\bigcup_{k=1}^{n} \bigcup_{l=1}^{n} B k l
$$

A vertical strip is the union of the $n$ sub-images $B_{k l}$ with the same value of $k$ (eq. 2), while a horizontal strip is the union of the $n$ sub-images $B_{k l}$ with the same value of $l$ (eq. 3):

$$
\begin{aligned}
& B^{V} k=\bigcup_{l=1}^{n} B k l \\
& B^{H} l=\bigcup_{k=1}^{n} B k l
\end{aligned}
$$

For each sector and strip (horizontal and vertical), the number of foreground pixels is counted. The maximum and minimum values are registered for vertical strips $\left(V S_{\max }\right.$, $\left.V S_{\min }\right)$, horizontal strips $\left(H S_{\max }, H S_{\min }\right)$, and for individual sub-images or sectors $\left(S_{\max }, S_{\min }\right)$. The vertical homogeneity index $\left(H_{n}^{V}\right)$ is computed using equation 4 , the horizontal homogeneity index $\left(H^{H}\right)$ with equation 5, and the sector homogeneity index $\left(H^{S}\right)$ with equation 6 . These indices vary between 0 and 100, with lower values corresponding to homogeneous cases. Values of 0 will occur when all strips or sectors have exactly the same number of foreground pixels, and values of 100 will occur when there is a void strip or sector, which is more likely to happen for large values of $n$ :

$$
\begin{gathered}
H^{V}{ }_{n}=\frac{V S_{\max }-V S_{\min }}{V S_{\max }+V S_{\min }} \times 100 \\
H^{H}{ }_{n}=\frac{H S_{\max }-H S_{\min }}{H S_{\max }+H S_{\min }} \times 100 \\
H^{S}=\frac{S_{\max }-S_{\min }}{S_{\max }+S_{\text {min }}} \times 100
\end{gathered}
$$

The values of the homogeneity indices vary with the scale parameter $n$. An average homogeneity index $\left\langle H^{H V S}{ }_{n}\right\rangle$ can be computed (for $H, V$, or $S$ ), using the $n-1$ individual indices (2 to $n$ ), as indicated in equation 7. This is a weighted average, with the lower values of $n$ contributing more to the average index:

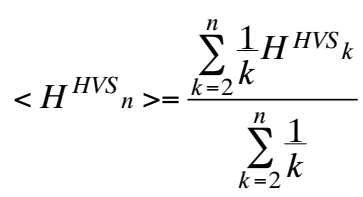

\section{Spot Size Spectra}

The spot size range and distribution can be computed from the binary images using image processing mathematical morphology. There are generally two possible approaches: (1) to use the standard grain counting method, which assumes that there is no overlap between drops, or (2) to use an overlapping model that accounts for multiple overlap between circular-shaped grains or droplets. Both methods use the elementary morphological operators dilation $(\oplus)$ and erosion $(\varnothing)$. The erosion and dilation operators use a structuring element $E$ (for example, a disk), which is usually of much smaller size than the image $I$. The dilation of $I$ by $E$ is $I \oplus E$, and the erosion of $I$ by $E$ is $I \varnothing E$. The morphological operator opening $(\Phi)$ (of $I$ by $E$ ) is defined as an erosion followed by a dilation, while the operator closing $(\Theta)$ (of $I$ by $E$ ) is defined as a dilation followed by an erosion (Gonzalez and Woods, 2002).

\section{Standard Method}

Morphological granulometries are performed by opening an image with increasing structuring elements in order to successively diminish the image (Dougherty and Sand, 1995). During granulometry, the image is successively sieved with a family of homothetic disks of increasing diameters (Prodanov et al., 2006). The process works by producing a set of images $I_{i}=I \Phi E_{i}$, where the structuring element $E_{i}$ is a disk of radius $i$. The object (or foreground) area is computed for each binary image $I_{i}$. The difference in object area between images $I_{i+1}$ and $I_{i}$ indicates the decrease in isolated objects of a similar size to the structuring element $E_{i}$. Dividing the area decrease by the area of the structuring element $E_{i}$ provides an estimate of the number of objects with the shape and size of $E_{i}$. This approach is effective for nonoverlapping grains, but when two grains overlap, there is a 
decrease in overall area, which is reflected in an incorrect evaluation of the number of grains and their sizes.

\section{Overlapping Method}

Instead of counting the decrease in area after each opening operation, the overlapping method uses an alternative approach. The original image $I$ is also subjected to morphological operations using structuring elements $E_{i}$ in the form of disks with radius $i$. However, the morphologic operation erosion is used instead of opening, resulting in images $I_{i}=I \varnothing E_{i}$. For each of these images, the number of 4-connected objects is computed, using the Matlab implementation (Mathworks, 2002) of the general procedure described by Haralick and Shapiro (1992). All pixels of each object identified by this procedure are linked to each other by a 4-neighborhood (top, bottom, left, right). The number of objects in each image $I_{i+1}$ is compared with the number of objects in image $I_{i}$, and the difference is assumed to be the number of circular objects of the size of the structuring element $E_{i}$. The process is repeated for circular structuring elements of increased size until there is no area left in the image. This method was validated with good results using synthetic images of various characteristics.

\section{Droplet Data Analysis}

The percentage of the image area covered with spots, the number of spots per target, and droplet size spectra factors were calculated for each WSP for different image resolutions, using the standard and overlapping methods.

The droplet spread on the WSP varies with physical properties such as surface tension, direction (angle), and energy of impact on the cards. The spread factors used to convert droplet stains on the WSP to actual droplet size, which cause the stain, are presented in table 1 (Syngenta, 2002). Using the spread factor conversion, it is possible to determine the diameter and volume of the original droplet that splashed on the WSP. A spread factor of 1.7 was used for stains of $100 \mu \mathrm{m}$ diameter or less, a spread factor of 2.1 for stains with diameter of $500 \mu \mathrm{m}$ or more, and a linear interpolation between the values presented in table 1 was used for stains with diameter between 100 and $500 \mu \mathrm{m}$.

Droplet size spectra factors corresponding to $10 \%\left(\mathrm{Dv}_{0.1}\right)$, $50 \%\left(\mathrm{Dv}_{0.5}\right)$, and $90 \%\left(\mathrm{D}_{\mathrm{v} 0.9}\right)$ of the cumulative spray liquid volume contained in the droplets up to the indicated diameter (ASAE Standards, 1997) are generally used to describe droplet spectra (Agüera et al., 2006). The ASAE standard droplet size spectra factors can be calculated once the droplet size spectra are obtained by the image processing techniques proposed.

Table 1. Spread factor values used to convert stain to droplet sizes (Syngenta, 2002).

\begin{tabular}{ccc}
\hline $\begin{array}{c}\text { Stain Diameter } \\
\text { of Drops }(\mu \mathrm{m})\end{array}$ & $\begin{array}{c}\text { Spread } \\
\left.\text { Factor }^{[\mathrm{a}}\right]\end{array}$ & $\begin{array}{c}\text { Droplet } \\
\text { Diameter }(\mu \mathrm{m})\end{array}$ \\
\hline 100 & 1.7 & 58.8 \\
200 & 1.8 & 111.1 \\
300 & 1.9 & 157.9 \\
400 & 2.0 & 200.0 \\
500 & 2.1 & 238.1 \\
\hline
\end{tabular}

[a] Assessed by the magnesium oxide and the silicon-oil-method at $20^{\circ} \mathrm{C}$, relative humidity of about $40 \%$ and the droplets reaching the WSP at sedimentation velocity.

\section{Manual Validation}

A manual counting of the number and size of stains was performed for sample S1 by visual inspection. The image obtained at 1200 dpi was printed in a plotter and magnified by a factor of 10 , resulting in an image poster of $51.22 \times$ $74.95 \mathrm{~cm}$. Each stain was measured with a ruler and labeled according to its size. The minimum diameter considered in the printed image was $1 \mathrm{~mm}$, corresponding to a stain radius of $0.05 \mathrm{~mm}$ in the original WSP. Linear regressions were established between manual and automatic counting data.

\section{RESULTS}

The algorithms and methods described in the previous section were applied to the three test samples (S1, S2, and S3) presented in figure 1. Image $\mathrm{S} 0$ was only used for validation purposes, and thus the results for that sample are only presented when they are thought to be relevant.

\section{Covered Area}

Once the color digital images are converted to binary images, the calculation of the area covered by spray is straightforward. The fraction of spray coverage for each of the test samples and scanning resolutions are presented in table 2. As it can be seen in the table, there is very little variability in the fraction of spray coverage counted with the scanning resolution.

\section{HOMOGENEITY INDICES}

The homogeneity indices were computed for the test samples for various values of the scale parameter $n$. The results are presented in table 3 for samples S1, S2, and S3. The homogeneity indices were computed for all image resolutions, but the results presented in this section only refer to the images at $600 \mathrm{dpi}$, as the variability of the homogeneity indices with the scanning resolution was found to be rather low (less than 5\%).

The homogeneity indices $H_{n}^{V}, H_{n}^{H}$, and $H_{n}^{S}$, presented in table 3 for values of $n$ between 2 and 7 permit an evaluation of the spatial homogeneity of the liquid spread on the test samples. S3 is the most homogeneous sample overall, while S2 has the highest values of $H^{V}, H^{H}$, and $H^{S}$ and thus shows less homogeneity horizontally, vertically, and in sectors. $\mathrm{S} 1$ is more homogeneous in the vertical than in the horizontal direction, and the opposite happens for S2 and S3. The values of $H^{V}$ are quite high for $\mathrm{S} 2$, which means that the amount of liquid spread varies considerably vertically. This can be easily confirmed by a visual inspection of the image (fig. 1). The average homogeneity indices for $n=10, n=20$, and $n=$ 50 are also presented in table 3 and show the weighting effect where the lower $n$ values contribute more to the average value reported.

An alternative way to analyze the homogeneity of a sample using the proposed homogeneity indices is to produce

Table 2. Fraction of spray coverage for test samples S0, S1, S2, and S3.

\begin{tabular}{ccccc}
\hline dpi & S0 (\%) & S1 (\%) & S2 (\%) & S3 (\%) \\
\hline 200 & 0.0 & 6.8 & 44.4 & 49.0 \\
300 & 0.0 & 7.3 & 44.6 & 49.8 \\
600 & 0.0 & 7.5 & 44.5 & 50.0 \\
1200 & 0.0 & 7.5 & 44.6 & 50.4 \\
\hline
\end{tabular}


Table 3. Homogeneity indices for samples S1, S2, and S3 (at 600 dpi).

\begin{tabular}{|c|c|c|c|c|c|c|c|c|c|}
\hline \multirow[b]{2}{*}{$n$} & \multicolumn{3}{|c|}{ S1 } & \multicolumn{3}{|c|}{ S2 } & \multicolumn{3}{|c|}{ S3 } \\
\hline & $H$ & $V$ & $S$ & $H$ & $V$ & $S$ & $H$ & $V$ & $S$ \\
\hline 2 & 12.14 & 0.23 & 18.91 & 16.27 & 29.31 & 45.74 & 0.18 & 1.02 & 8.91 \\
\hline 3 & 15.81 & 8.34 & 27.96 & 19.43 & 38.50 & 53.14 & 5.57 & 11.51 & 23.81 \\
\hline 4 & 18.95 & 3.75 & 37.02 & 23.31 & 44.97 & 66.47 & 7.01 & 12.98 & 32.98 \\
\hline 5 & 22.84 & 16.85 & 54.88 & 24.55 & 48.33 & 74.38 & 8.71 & 15.32 & 36.25 \\
\hline 6 & 23.42 & 14.73 & 61.84 & 26.97 & 49.33 & 80.97 & 10.54 & 16.21 & 40.48 \\
\hline 7 & 25.69 & 17.91 & 72.98 & 24.98 & 53.82 & 84.87 & 9.16 & 16.55 & 41.54 \\
\hline$<\mathrm{H} 10>$ & 19.58 & 10.38 & 45.88 & 21.95 & 43.69 & 65.65 & 6.17 & 11.33 & 29.24 \\
\hline$<\mathrm{H} 20>$ & 25.04 & 17.27 & 59.54 & 25.11 & 48.99 & 72.96 & 8.22 & 14.78 & 40.61 \\
\hline$<\mathrm{H} 50>$ & 31.85 & 30.15 & 69.96 & 28.72 & 53.72 & 79.93 & 10.75 & 18.84 & 54.28 \\
\hline
\end{tabular}

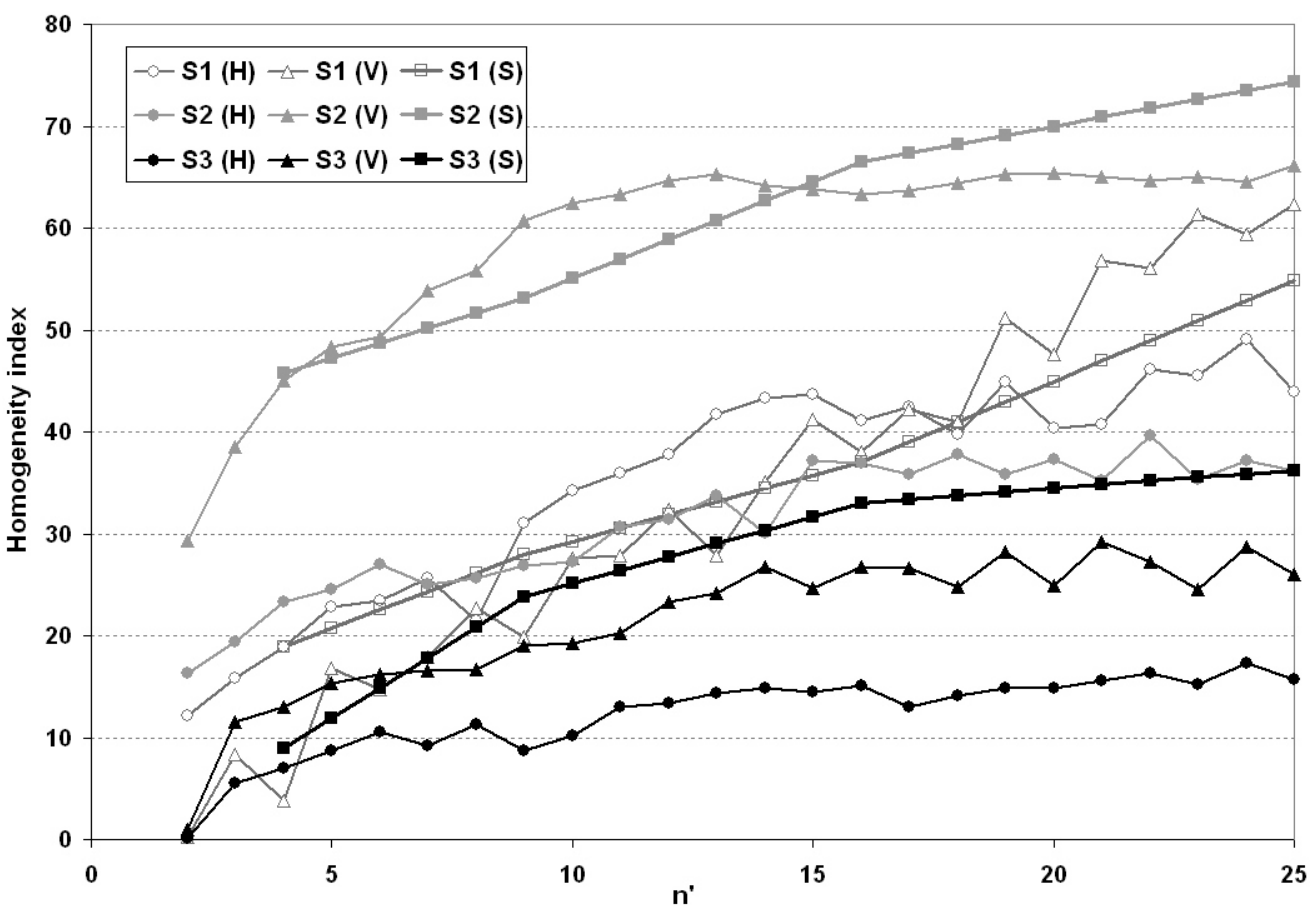

Figure 3. Homogeneity indices (horizontal, vertical, and sector) versus normalized scaling parameter $\boldsymbol{n}^{\prime}$.

a plot of $H_{n}^{V}, H_{n}^{H}$, and $H_{n}^{S}$ as a function of the scaling parameter $n$. Perhaps a more effective way to compare the results from the sector homogeneity index and the other indices is to use a normalization factor $n^{\prime}$, which ensures that both sectors and strips have the same area. For the sector indices, a value of $n=2$ will correspond to $n^{\prime}=4, n=3$ to $n^{\prime}=$ 9 , and so forth $\left(n^{\prime}=n^{2}\right)$, while for the strip indices $n^{\prime}=n$. Such a plot is presented in figure 3 for the three test samples. As expected, there is a general tendency for the homogeneity indices to increase with $n^{\prime}$.

The homogeneity index data presented in figure 3 clearly reveal the much higher homogeneity of S3, which remains low as the scaling parameter increases (low index values correspond to homogeneous cases). S1 is also homogeneous for low values of $n^{\prime}$, but the indices for S1 increase rather rapidly with increasing $n^{\prime}$. For S2 there are generally higher values than for the other samples, except for the horizontal homogeneity that remains reasonably low, even for large values of $n^{\prime}$.

\section{Stain Size Spectra}

The stain size range and distribution were computed for all test samples and resolutions using both the standard and the overlapping methods. The results for the 600 dpi images are presented in figure 4 for the standard method and in figure 5 for the overlapping method. The plots produced by the two methods are very different, particularly regarding the number of small-sized stains. The plot for S3 produced using the standard method is not fully represented in figure 4 , as it peaks at 1126 stains for radius $0.042 \mathrm{~mm}$.

The results produced from the standard and overlapping methods are presented together in figure 6 for sample 1 data obtained at 300, 600, and $1200 \mathrm{dpi}$. Again, it is clear from these combined plots that the differences between the two methods are mostly noticeable for stains with small radius. This is due to the fact that the standard method does not account for overlap between stains. For example, when two equal-sized stains overlap, the morphological operation opening with a small-sized disk will result in a reduction of the covered area. This will be wrongly counted as a number of small-sized stains, which in fact are not present in the image. The result is an overestimation of the number of small stains when the standard method is used in an overlapping scenario. 


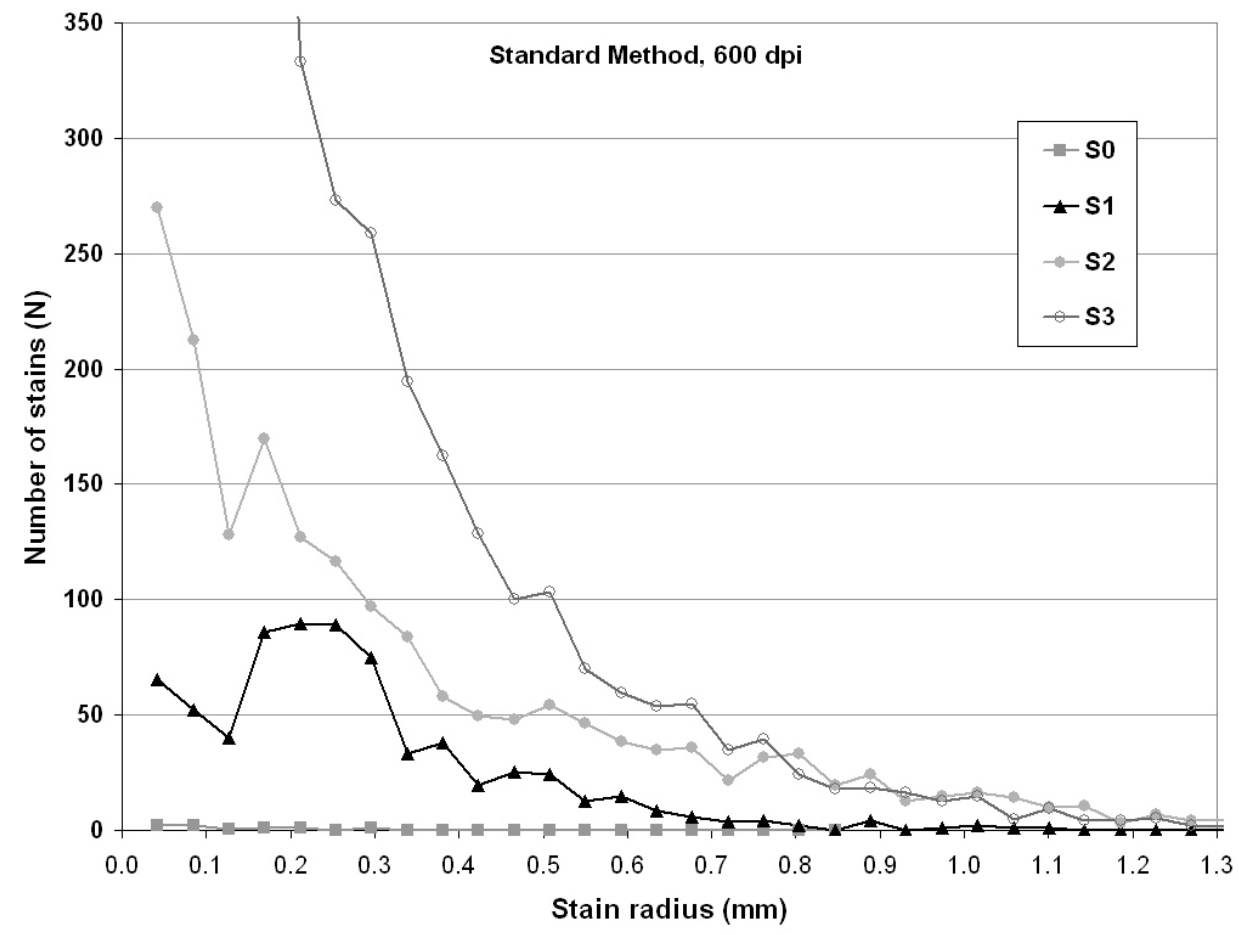

Figure 4. Stain size spectra for the four test samples at 600 dpi using the standard model.

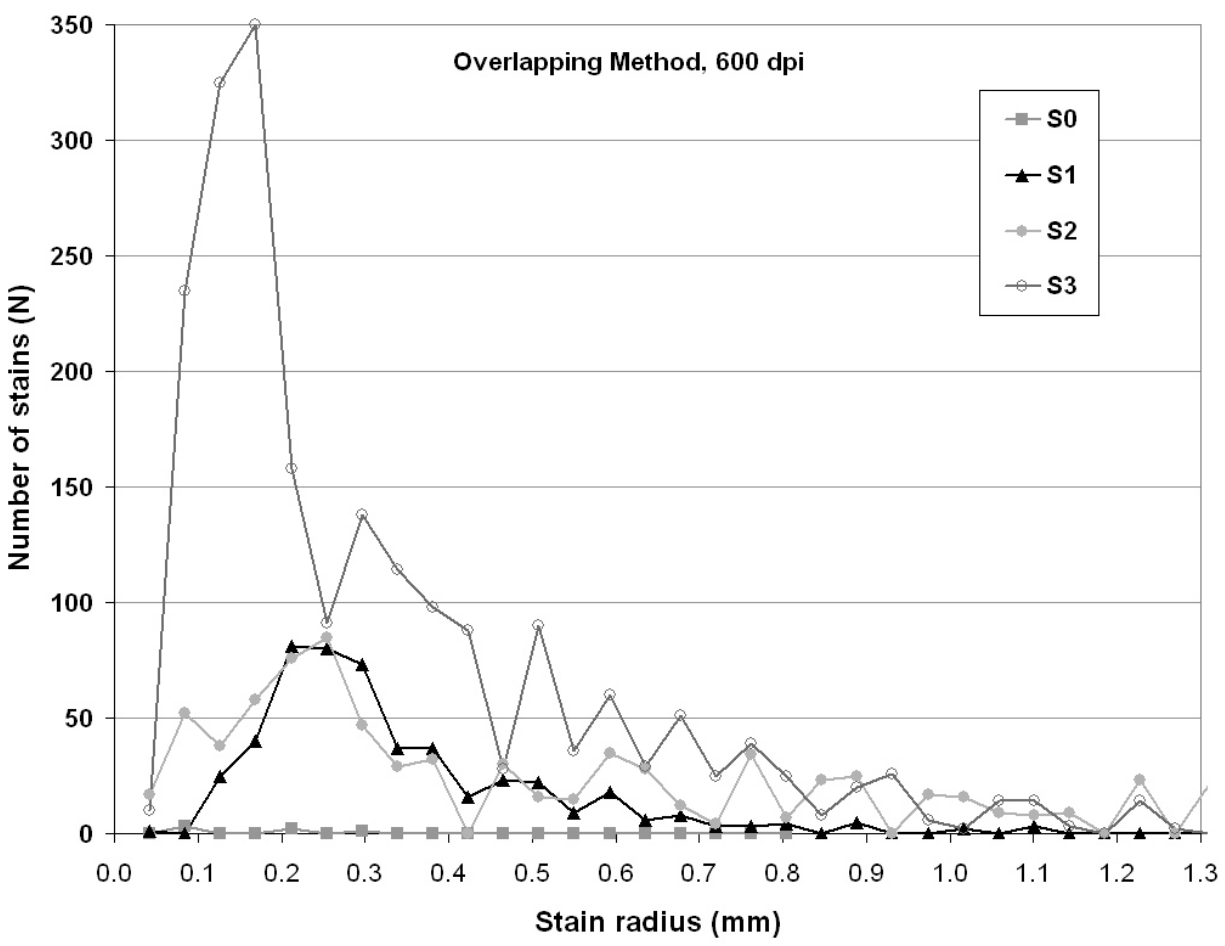

Figure 5. Stain size spectra for the four test samples at 600 dpi using the overlapping model.

\section{Evaluation of the Scanning Resolution Effect}

An evaluation of the effect of the scanning resolution on the computation of the stain size range and distribution was carried out. Equivalent plots were produced for each sample and scanning resolution (200, 300, 600, and $1200 \mathrm{dpi})$ using the data obtained from the four different scanning resolutions interpolated or down-sampled to the reference resolution. An example of this analysis is presented in figure 7 for test image
S1 and reference scanning resolution of $600 \mathrm{dpi}$. The stain size range and distribution profiles obtained from the 200 and 300 dpi data were linearly interpolated to the reference resolution, and the profile obtained from the 1200 dpi data was down-sampled to $600 \mathrm{dpi}$, by averaging every two sampled values. The plot indicates that there is a sharper evaluation of the stain size using the higher resolution data, particularly for small-sized stains. The various curves tend to 


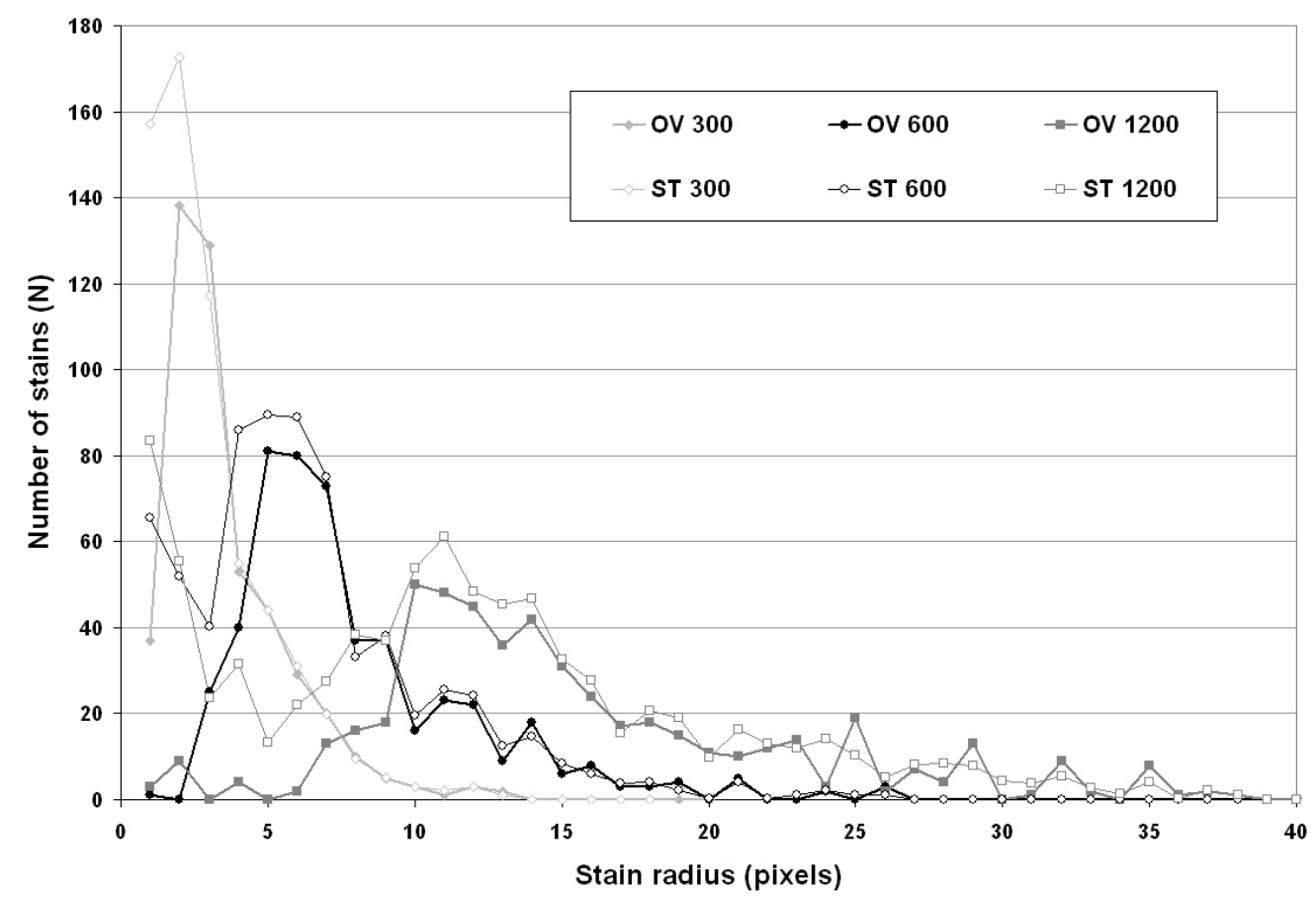

Figure 6. Stain size spectra plots for sample S1 obtained using the standard (ST) and overlapping (OV) methods at 300, 600, and 1200 dpi.

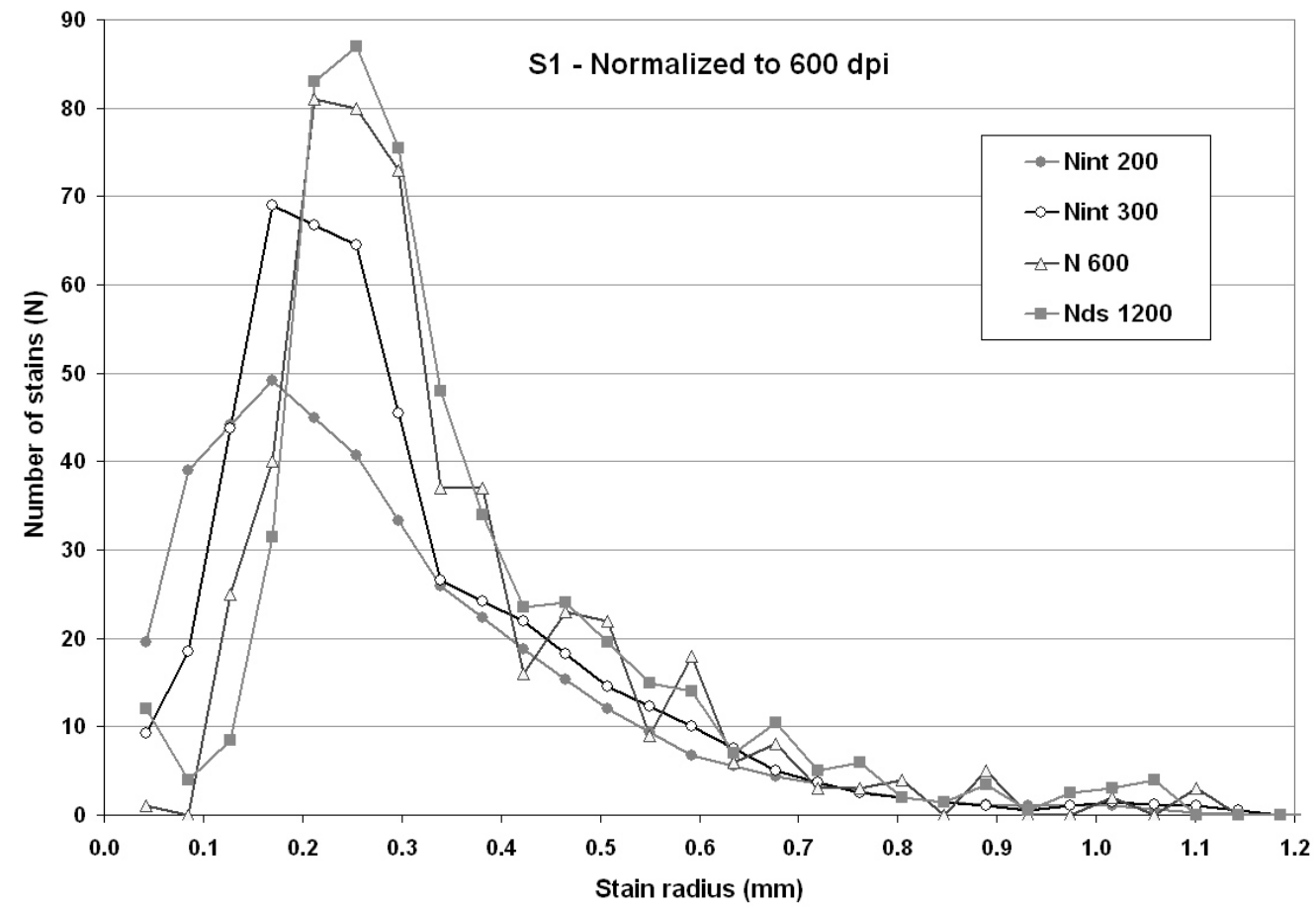

Figure 7. Stain size spectra for test sample S1 obtained from data of 200, 300, 600, and $1200 \mathrm{dpi}$, all normalized to $600 \mathrm{dpi}$.

follow a similar trend for large radius values. The profiles for 200 and $300 \mathrm{dpi}$ are smoother, as $1 / 3$ and $1 / 2$ of the points in these plots were obtained by linear interpolation. The $600 \mathrm{dpi}$ resolution seems to be the most suitable choice, as the stain profiles are very close to the $1200 \mathrm{dpi}$ profiles but the computational load is much lower. Furthermore, very high scanning resolution rates tend to introduce unwanted artifacts.

\section{Number of Droplets Per Unit Area}

The number of droplets per unit area $\left(\mathrm{cm}^{2}\right)$ was computed for each sample and scanning resolution tested. The results are presented in table 4 using the overlapping model. The number of droplets per unit area computed using the standard method is less reliable, as this method tends to largely overestimate the number of small-sized droplets. The values computed for each sample increase with the scanning resolution, as there is an increase of the number of droplets counted, particularly for small-sized droplets. 
Table 4. Number of droplets per unit area $\left(\mathrm{cm}^{2}\right)$ for test samples S0, S1, S2, and S3.

\begin{tabular}{ccccc}
\hline dpi & S0 & S1 & S2 & S3 \\
\hline 200 & 0.05 & 10.59 & 12.45 & 25.42 \\
300 & 0.08 & 12.35 & 16.69 & 36.99 \\
600 & 0.16 & 12.92 & 22.38 & 56.16 \\
1200 & 0.50 & 13.67 & 28.22 & 72.23 \\
\hline
\end{tabular}

\section{Droplet Size Spectra Factors}

The droplet size spectra were computed from the stain size spectra using the spread factors presented in table 1 (Syngenta, 2002). The values of the ASAE standard droplet size spectra factors were computed for all test samples and resolutions using the overlapping model. The values of the ASAE standard droplet size spectra factors were also computed using the standard method, but only for $600 \mathrm{dpi}$. The values of $\mathrm{Dv}_{0.1}, \mathrm{Dv}_{0.5}$, and $\mathrm{Dv}_{0.9}$ are presented in table 5 . The pixel size and minimum stain and droplet diameters that can be evaluated for each scanning resolution are also presented in table 5 .

The results obtained for the various scanning resolutions tested present consistent values, with differences between consecutive scanning resolutions generally smaller than the minimum drop diameter sampled. There is clearly a limitation in the computation of these parameters due to the discrete nature of the image data. As an illustration, figure 8 shows the fractional volume for sample S1 computed using the images scanned at 200, 300, 600, and 1200 dpi. The limitations of the lower resolution images can be clearly observed in this plot.

A comparison between the values computed using the overlapping and the standard methods indicates that the differences are not very large. Although the droplet profiles produced from both methods are very different, as can be seen in figures 4 through 6 , the values of $\mathrm{Dv}_{0.1}, \mathrm{Dv}_{0.5}$, and $\mathrm{Dv}_{0.9}$ are not as different as might be expected from an inspection of these plots, as the contribution of small-sized droplets in terms of volume is rather small. For S1, the values of $\mathrm{Dv}_{0.1}, \mathrm{Dv}_{0.5}$ are the same, and there is only a small
Table 5. Droplet size spectra factors $D_{10}, D_{50}$, and $D_{90}(\mathrm{~mm})$ for the test samples S1, S2, and S3.

\begin{tabular}{|c|c|c|c|c|c|}
\hline \multirow{3}{*}{$\begin{array}{c}\text { Factor } \\
(\mathrm{mm})\end{array}$} & \multicolumn{4}{|c|}{ Overlapping Method } & \multirow{3}{*}{$\begin{array}{c}\begin{array}{c}\text { Standard } \\
\text { Method }\end{array} \\
600 \mathrm{dpi}\end{array}$} \\
\hline & \multirow{2}{*}{$\begin{array}{l}200 \\
\text { dpi }\end{array}$} & \multirow{2}{*}{$\begin{array}{l}300 \\
\text { dpi }\end{array}$} & \multirow{2}{*}{$\begin{array}{l}600 \\
\text { dpi }\end{array}$} & \multirow{2}{*}{$\begin{array}{c}1200 \\
\text { dpi }\end{array}$} & \\
\hline & & & & & \\
\hline Pixel size & 0.13 & 0.08 & 0.04 & 0.02 & 0.04 \\
\hline Min. stain dia. & 0.25 & 0.17 & 0.08 & 0.04 & 0.08 \\
\hline Min. droplet dia. & 0.15 & 0.10 & 0.05 & 0.02 & 0.05 \\
\hline $\mathrm{S} 1-\mathrm{Dv}_{0.1}$ & 0.24 & 0.32 & 0.28 & 0.30 & 0.28 \\
\hline $\mathrm{S} 1-\mathrm{Dv}_{0.5}$ & 0.60 & 0.56 & 0.56 & 0.65 & 0.56 \\
\hline $\mathrm{S} 1-\mathrm{Dv}_{0.9}$ & 0.97 & 0.97 & 1.05 & 1.01 & 0.97 \\
\hline $\mathrm{S} 2-\mathrm{Dv}_{0.1}$ & 0.85 & 0.81 & 0.93 & 0.95 & 0.60 \\
\hline $\mathrm{S} 2-\mathrm{Dv}_{0.5}$ & 1.94 & 1.85 & 1.81 & 1.89 & 1.29 \\
\hline $\mathrm{S} 2-\mathrm{Dv}_{0.9}$ & 2.54 & 2.74 & 2.38 & 2.36 & 2.34 \\
\hline $\mathrm{S} 3-\mathrm{Dv}_{0.1}$ & 0.48 & 0.48 & 0.48 & 0.44 & 0.36 \\
\hline $\mathrm{S} 3-\mathrm{Dv}_{0.5}$ & 0.97 & 0.89 & 0.89 & 0.91 & 0.77 \\
\hline $\mathrm{S} 3-\mathrm{Dv}_{0.9}$ & 1.69 & 1.61 & 1.49 & 1.63 & 1.37 \\
\hline
\end{tabular}

difference for $\mathrm{Dv}_{0.9}$. For $\mathrm{S} 2$ and $\mathrm{S} 3$, the differences in the standard droplet size spectra factors computed by the two methods are higher, particularly as to what concerns $\mathrm{Dv}_{0.1}$. This is due to the overestimation of small-sized droplets by the standard method.

\section{Manual Validation}

Manual counting of the number and size of stains was performed for sample S1 by visual inspection. The manual identification and labeling of stains is a laborious and difficult task, and the results produced cannot be considered absolutely accurate. The main difficulties are related to assigning a size to non-circular stains, and also in areas with multiple overlap between stains. The comparison between the automatic and manual measurements is also difficult, as the minimum size increment varies: $0.127 \mathrm{~mm}$ (for $200 \mathrm{dpi}$ ), $0.085 \mathrm{~mm}$ (300 dpi), $0.042 \mathrm{~mm}(600 \mathrm{dpi})$, and $0.021 \mathrm{~mm}$ (1200 dpi) for automatic counting, and $0.050 \mathrm{~mm}$ for manual counting. The number of stains measured by each method, manual and automatic (overlapping and standard), were nonetheless compared by setting up a common range of stain

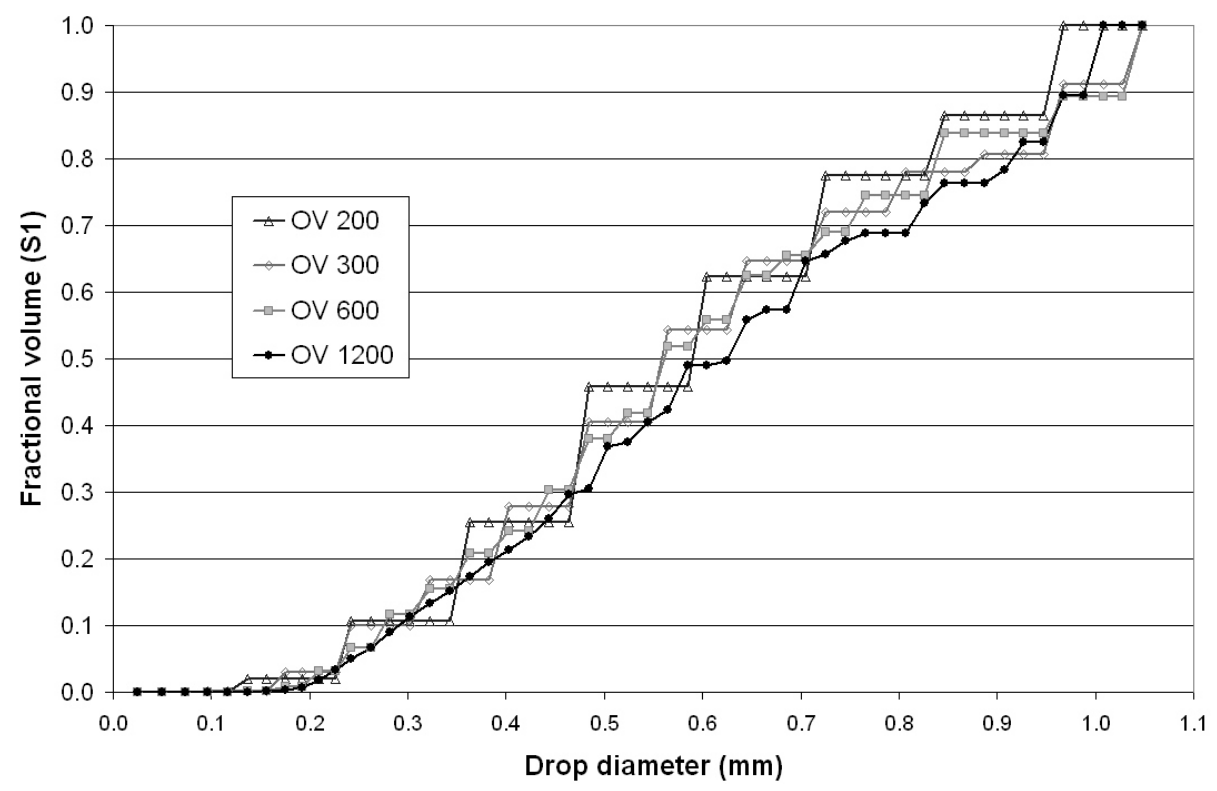

Figure 8. Fractional volume for test sample $\mathrm{S1}$ computed with droplet profiles obtained by the overlapping method (OV) and image data scanned at $200,300,600$, and $1200 \mathrm{dpi}$. 
Table 6. Number of stains counted manually and by the automatic methods (ST and OV) for sample S1, and summary of regression results: coefficient of determination $\left(R^{2}\right)$ and statistical significance.

\begin{tabular}{|c|c|c|c|c|c|c|c|}
\hline \multirow{2}{*}{$\begin{array}{l}\text { Stain Radius } \\
\qquad(\mu \mathrm{m})\end{array}$} & \multirow{2}{*}{$\begin{array}{l}\text { Manual } \\
\text { Counting }\end{array}$} & \multicolumn{3}{|c|}{ Overlapping Method } & \multicolumn{3}{|c|}{ Standard Method } \\
\hline & & OV300 & OV600 & OV1200 & ST300 & ST600 & ST1200 \\
\hline$<176$ & 25 & 175 & 66 & 47 & 329.7 & 243.6 & 295.0 \\
\hline 176 to 275 & 199 & 129 & 161 & 197 & 117.1 & 176.3 & 245.5 \\
\hline 276 to 375 & 153 & 53 & 110 & 114 & 55.0 & 108.1 & 122.5 \\
\hline 376 to 475 & 66 & 44 & 76 & 66 & 44.1 & 83.3 & 78.8 \\
\hline 476 to 575 & 47 & 29 & 31 & 45 & 30.9 & 36.8 & 49.8 \\
\hline 576 to 675 & 21 & 20 & 24 & 18 & 19.9 & 23.1 & 24.5 \\
\hline 676 to 775 & 12 & 15 & 14 & 20 & 14.7 & 13.8 & 14.1 \\
\hline 776 to 875 & 4 & 3 & 4 & 6 & 3.0 & 2.4 & 6.6 \\
\hline 876 to 975 & 6 & 1 & 5 & 5 & 2.2 & 5.4 & 2.4 \\
\hline$>975$ & 9 & 5 & 5 & 7 & 4.0 & 4.2 & 3.3 \\
\hline Total & 766 & 778 & 723 & 769 & 1067.4 & 1120.9 & 1383.0 \\
\hline $\mathrm{R}^{2}$ & & 0.266 & 0.910 & 0.958 & 0.050 & 0.335 & 0.368 \\
\hline Significance & & ns & 0.000 & 0.000 & ns & ns & 0.048 \\
\hline
\end{tabular}

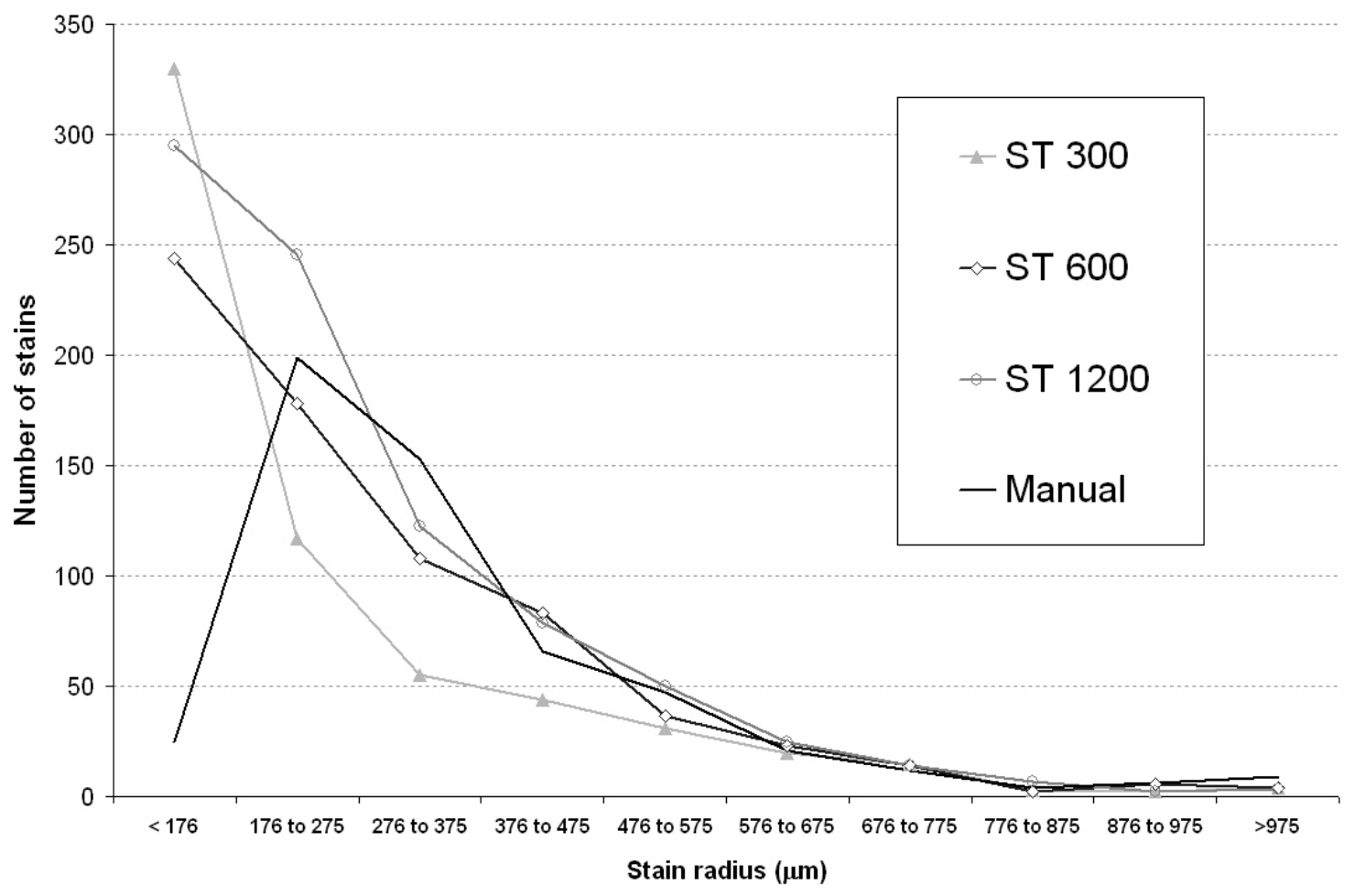

Figure 9. Number of stains counted manually and by the standard method (at 300, 600, and 1200 dpi) for test sample S1.

radius. The results are presented in table 6 and in figures 9 and 10. The total number of stains counted manually and by the proposed overlapping method are reasonably close, although the size distribution does not exactly match. The performance of the standard method is much worse because the total number of stains is largely overestimated, mainly due to the excessive number of small stains that were wrongly counted.

Linear regressions were established between the manual and automatic counting data. The coefficient of determination $\left(\mathrm{R}^{2}\right)$ for the linear regression and the statistical significance are presented in table 6 . The values of $\mathrm{R}^{2}$ are high for the overlapping methods at 600 and $1200 \mathrm{dpi}(0.910$ and 0.958 , respectively).

The stain sizes counted manually were converted to droplet sizes using the spread factors proposed by Syngenta (2002). The resulting droplet profiles were used to compute the droplet size spectra factors corresponding to $10 \%\left(\mathrm{Dv}_{0.1}\right)$, $50 \%\left(\mathrm{Dv}_{0.5}\right)$, and $90 \%\left(\mathrm{Dv}_{0.9}\right)$ of the cumulative spray liquid volume. The results obtained were: $\mathrm{Dv}_{0.1}=0.29, \mathrm{Dv}_{0.5}=$ 0.62 , and $\mathrm{Dv}_{0.9}=1.09$. These values are very close to those obtained using the automatic methods presented in table 5, particularly for the overlapping method using 600 and 1200 dpi images.

\section{Discussion AND Conclusion}

The fully automatic methodology proposed, based on image processing of scanned WSP, provides a number of parameters to characterize the spray: (1) fraction of spray coverage, (2) homogeneity parameters, (3) stain and droplet number and size distribution profiles, and (4) standard droplet size spectra factors. The algorithms and methods 


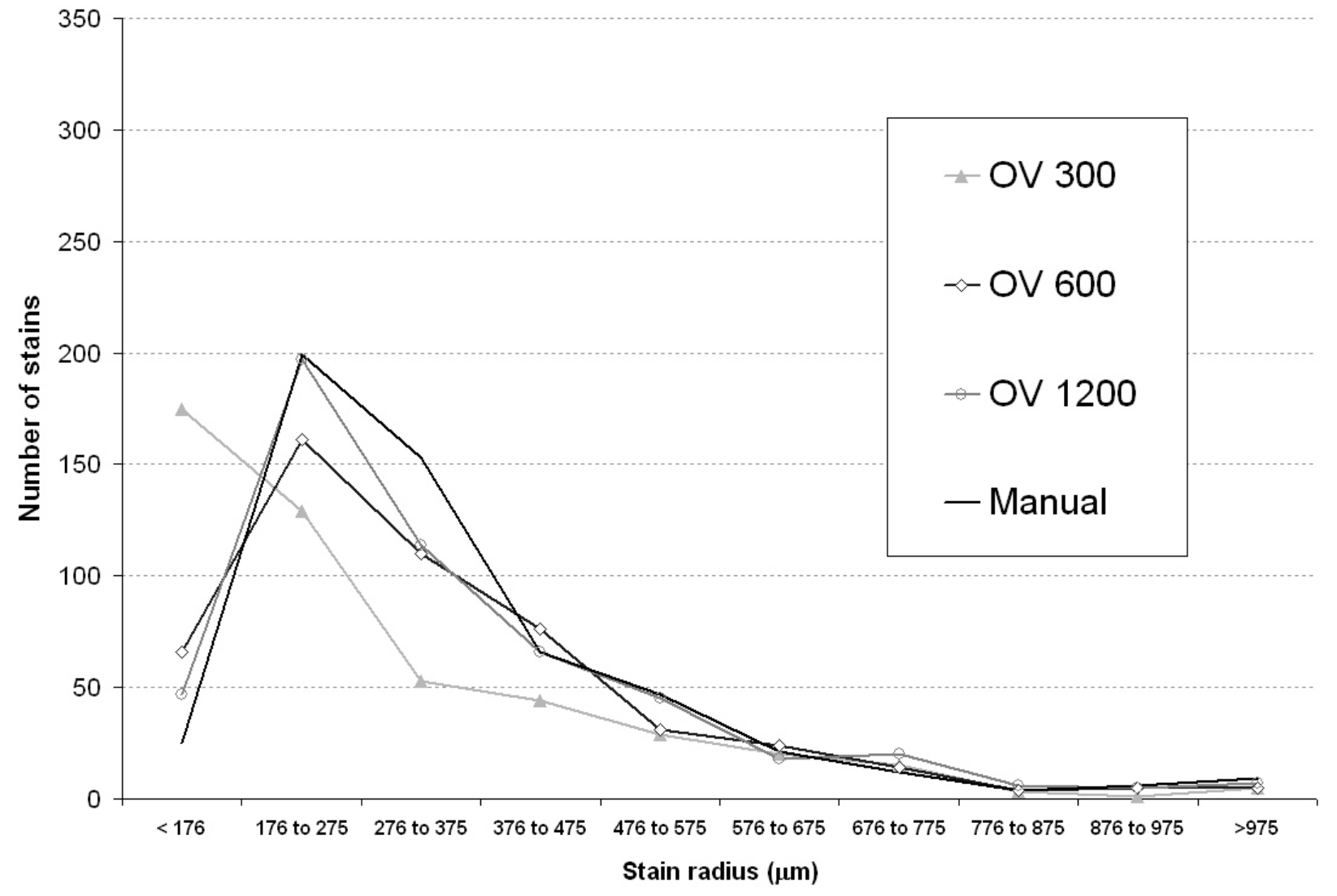

Figure 10. Number of stains counted manually and by the overlapping method (at 300, 600 , and 1200 dpi) for test sample S1.

proposed are effective in reducing the main limitations resulting from the stain overlap, which are inevitably present in WSP using high spray coverage, although further testing is still required.

The algorithms developed in this study were used to calculate the fraction of spray coverage in WSP, ranging from $7 \%$ to $50 \%$. The proposed homogeneity indices were used to characterize the spatial homogeneity of the liquid spread at various scales. The homogeneity indices can be used for evaluation of spray quality from herbicide application at bare soil in different crop systems (e.g., orchard tree rows maintained as bare soil by herbicide sprays, or no-tillage crop systems). No significant differences were found in the computation of both the fraction of spray coverage and the homogeneity indices for the different scanning resolutions tested.

The proposed method for obtaining the droplet size spectra and the droplet size spectra factors seems to perform rather well when the number of droplets is high. A comparison between the values of the standard droplet size spectra computed using the overlapping and standard methods indicated an overestimation of small-sized droplets computed by the standard method. This is a critical point for spray evaluation of new and modified pesticides and improved application techniques with low volumes and coarse droplets spectra. The proposed overlapping method is therefore more adequate for this particular problem, providing more accurate results than the standard method. However, it is worth noting that when two droplets fully overlap, the method is unable to distinguish them from a single droplet. There are some noticeable differences for the droplet size spectra extracted from the various scanning resolutions, with more detailed information extracted from higher resolution data, but the effect of the scanning resolution on the standard droplet size spectra factors is not very large. As the image processing morphologic operations are computationally heavy, a tradeoff between accuracy and speed has to be made. The $600 \mathrm{dpi}$ scanning resolution was found to be the best choice according to these two factors.

Experimental data have indicated that the WSP, for appropriate scanning resolutions, can provide good results not only in terms of stains size spectra factors but also concerning the homogeneity of coverage. The method proposed for counting overlapping droplets based on mathematical morphology proved to be quite effective for spray evaluation of WSP converted to binary images. This software was developed specifically for the analysis of WSP and can, in the future, be delivered to farmers to provide an easy, fast, automated, and accurate analysis of the spray quality, minimizing human error. A future development of this work might be a portable scanner for image capture in the field, with the developed algorithms incorporated.

\section{ACKNOWLEDGEMENTS}

This work was done with the support of Centro de Investigação em Ciências Geo-Espaciais, Faculdade de Ciências da Universidade do Porto, financed by the Portuguese National Science Foundation (FCT-Fundação para a Ciência e a Tecnologia). The authors wish to thank Arlete Rodrigues for the manual counting of stains. 


\section{REFERENCES}

AgroScan. 2008. Análise de gotas em pulverizações agrícolas utilizando digitalização de imagens. Pelotas, Brazil: Agrotec. Available at: www.agrotec.etc.br/produtos/agroscan. Accessed 13 March 2008.

Agüera, F., F. J. Aguilar, M. A. Aguilar, and F. Carvajal. 2006. Atomization characteristics of hydraulic nozzles using fractal geometry. Trans. ASAE 49(3): 581-587.

ASAE Standards. 1997. S.327.2: Terminology and definitions for agricultural chemical application. St. Joseph, Mich. ASAE.

Bateman, R. 1993. Simple, standardized methods for recording droplet measurements and estimation of deposits from controlled droplet applications. Crop Protection 12(3): 201-206.

Crowe, T. G., D. Downey, and D. K. Giles. 2005. Digital device and technique for sensing distribution of spray deposition. Trans. ASAE 48(6): 2085-2093.

Dougherty, E. R., and F. Sand. 1995. Representation of linear granulometric moments for deterministic and random binary Euclidean images. J. Visual Comm. Image Rep. 6(1): 69-79.

Fox, R. D., R. C. Derksen, H. E. Ozkan, and R. D. Brazee. 1998. Methods and techniques in determining spray quality. In Proc. Conf. on Measurement and Management of Agrochemical Spray Quality, 123-138. Taichung, Taiwan: Taiwan Agricultural Research Institute.

Fox, R. D., R. C. Derksen, J. A. Cooper, C. R. Krause, and H. E. Ozkan. 2003. Visual and image system measurement of spray deposits using water-sensitive paper. Applied Eng. in Agric. 19(5): 549-552.

Giles, D. K., and D. Downey. 2003. Quality control verification and mapping for chemical application. Precision Agric. 4(1): 103-124.

Gonzalez, R. C., and R. E. Woods. 2002. Digital Image Processing. Englewood Cliffs, N.J.: Prentice Hall.

Haralick, R. M., and L. G. Shapiro. 1992. Computer and Robot Vision, 28-48. Vol. I. Boston, Mass.: Addison-Wesley.
Hoffman, W. C. 2003. Field swath and drift analyses techniques. ASAE Paper No. AA03-007. St. Joseph, Mich.: ASAE.

Holownicki, R., G. Doruchowski, W. Swiechowski, and P. Jaeken. 2002. Methods of evaluation of spray deposit and coverage on artificial targets. Electronic J. Polish Agric. Universities 5(1): $1-9$.

Mathworks. 2002. Using Matlab. Ver 6.5. Natick, Mass.: The MathWorks, Inc.

Panneton, B. 2002. Image analysis of water-sensitive cards for spray coverage experiments. Applied Eng. in Agric. 18(2): 179-182.

Prodanov, D., J. Heeroma, and E. Marani. 2006. Automatic morphometry of synaptic boutons of cultured cells using granulometric analysis of digital images. J. Neuroscience Methods 151(2): 168-177.

Salyani, M., and R. D. Fox. 1999. Evaluation of spray quality by oil- and water-sensitive papers. Trans. ASAE 42(1): 37-43.

Syngenta. 2002. Water-sensitive paper for monitoring spray distributions. CH-4002. Basle, Switzerland: Syngenta Crop Protection.

Turner, C., and K. Huntington. 1970. The use of water-sensitive dye for the detection and assessment of small spray droplets. $J$. Agric. Eng. Res. 15(4): 385-387.

Wolf, R. E. 2003. Assessing the ability of DropletScan to analyze spray droplets from a ground operated sprayer. Applied Eng. in Agric. 19(5): 525-530.

UTHSCSA ImageTool. 2002. UTHSCSA ImageTool for Windows. Version 3.0. University of Texas Health Science Center San António (UTHSCSA). Available at: http://ddsdx.uthscsa.edu/dig/itdesc.html. Accessed 13 March 2008.

Zhu, H., R. C. Derksen, C. R. Krause, R. D. Fox, R. D. Brazee, and H. E. Ozkan. 2005. Fluorescent intensity of dye solutions under different $\mathrm{pH}$ conditions. J. ASTM Intl. 2(6): 2-7. 
